\title{
A survey about the contents and features of oncology clinical pharmacy services and self- evaluations of the oncology pharmacists in China
}

\section{Yan-Ting Wang}

National Cancer Center/National Clinical Research Center for Cancer/Cancer Hospital, Chinese Academy of Medical Sciences and Peking Union Medical College https://orcid.org/0000-0001-8584-2079

\section{Yuan-Yuan Dai}

National Cancer Center/National Clinical Research Center for Cancer/Cancer Hospital Chinese Academy of Meical Sciences and Peking Union Medical College

Jun Yang

National Cancer Center/National Clinical Research Center for Cancer/Cancer Hospital Chinese Academy of Medical Sciences and Peking Union Medical College

Hai-Yan Zhou

National Cancer Center/National Clinical Research Center for Cancer/Cancer Hospital Chinese Academy of Medical Sciences and Peking Union Medical College

\section{Zhe Chen}

National Cancer Center/National Clinical Research Center for Cancer/Cancer Hospital Chinese Academy of Medical Sciences and Peking Union Medical College

\section{Guo-Hui Li (D Igh0603@126.com )}

https://orcid.org/0000-0002-9112-4142

\section{Research article}

Keywords: Oncology, pharmacist, clinical pharmacy services, self-evaluation, survey, China

Posted Date: March 3rd, 2020

DOI: https://doi.org/10.21203/rs.3.rs-15705/v1

License: (9) This work is licensed under a Creative Commons Attribution 4.0 International License. Read Full License

Version of Record: A version of this preprint was published at Journal of Clinical Pharmacy and Therapeutics on June 26th, 2020. See the published version at https://doi.org/10.1111/jcpt.13191. 


\section{Abstract}

Background: With the growing number of oncology patients and novel anti-tumor medications, the demand of oncology clinical pharmacy services is increasing rapidly in China. However, the current status of oncology clinical pharmacy services in China were seldom reported. The purpose of the study was to investigate the contents and features of oncology clinical pharmacy services and self-evaluations of the oncology pharmacists in China, and to identify related problems for its further development.

Methods: A 55-item online questionnaire was distributed to oncology-related clinical pharmacists in Chinese hospitals in January 2019. Pharmacists answered the questions on the WeChat platform. Data were analyzed using descriptive statistics.

Results: The respondents of the 166 valid questionnaires were from 29 provinces (or autonomous regions or municipalities). Their average working experience for clinical pharmacy was 4.86 years. The average duration of their clinical services was 3.27 hours/day. The clinical pharmacy services consist of 15 types and their workload on each service type were identified. Pharmacists thought they were not good at establishing pharmacotherapy protocols, participating in therapeutic drug monitoring, pharmacy consultation and pharmacogenetic/pharmacogenomic service. About $80 \%(81.33 \%)$ of the pharmacists thought other healthcare professionals knew the nature of their work, and some services were very welcomed by clinical departments. The biggest barrier for career development of the pharmacists was insufficiency of professional skills. In most cases, the clinical service accounted for less than $30 \%$ of the overall performance evaluation of the pharmacists. Most of the participating pharmacists were not satisfied with their current salary. The overall evaluation score for their career wellbeing was 6.27 out of 10.

Conclusions: The value of oncology clinical pharmacists is being increasingly recognized, but advanced services need further expansion. Increasing the time serving in clinical units, optimizing performance evaluation and strengthening their skills are possible ways for pharmacists to provide superior services. This study showed the current service of oncology clinical pharmacists in most parts of China, which may provide reference for policy makers, promote international communications and shed light on future development of oncology clinical pharmacy in China.

Key words: Oncology, pharmacist, clinical pharmacy services, self-evaluation, survey, China

\section{Background}

In recent years, although the 5-year survival rates of most cancer types in China have been increasing, there is still a large gap between China and other developed countries [1]. Drug therapy is one of the pillars in cancer comprehensive therapy. However, due to the complexity of anti-tumor drugs and increased number of the new drug approvals, together with the complicated disease states of cancer patients (e.g. elderly patients with multiple comorbidities), cancer treatment in China still faces great challenges. 
Clinical pharmacists are important members in the multidisciplinary team of cancer therapy. In recent years, with the strong support from governmental departments, increased concern from the general public, and the rapid progress of oncology clinical pharmacy, clinical pharmacists are facing great opportunities in cancer treatment. In 2016, the National Health and Family Planning Commission and the State Administration of Traditional Chinese Medicine jointly issued the "Notice on Strengthening the Management of Standardized Diagnosis and Treatment of Tumors" ([2016] No. 7), which clearly stated that the training of oncology clinical pharmacists should be strengthened, and their ability to review, evaluate, and distribute anti-tumor medications and related adjuvant medications should be enhanced, so as to offer guidance for the clinical application of anti-tumor medications" [2]. In 2018, the National Health Commission of the People's Republic of China organized the experts of Rational Medication Use to lead the development and release of the "Guiding principles for the Clinical Application of New Anti-tumor Medications (2018 Edition)", which stated that the professional team for clinical application and management of anti-tumor medications should be composed of professionals from oncology internal medicine, oncology surgery, pathology, clinical pharmacy, imaging, etc., so as to provide technical guidance and consultation for the rational use of medications in clinical departments, and to provide medical staff and lower-level medical institutions with relevant information on the clinical application of anti-tumor medications" [3]. The Oncology Clinical Pharmacy Committee of the China Anti-Cancer Association has recently organized clinical pharmacy and medical experts all over China to develop and publish a series of expert consensuses on promoting the rational use of anti-tumor medications in clinical practice [4-6]. These all show that pharmacists are playing increasingly important roles in cancer treatment. However, little is known about the contents and features of the oncology clinical pharmacy services in China, and the self-evaluation of clinical pharmacists and the evaluation from other healthcare professionals are also rarely reported. Thus, a questionnaire was designed to investigate the current status of oncology pharmacy services in China, in order to identify and summarize related problems and to offer reference to optimizing the services in the future.

\section{Methods}

The aim, questionnaire design and setting of the study

The aim of the study was to investigate the contents and features of oncology clinical pharmacy services and self-evaluations of the oncology pharmacists in China, and to identify related problems for its further development. The setting of the study was in the hospitals.

The design of the questionnaire items was based on literature review, the general situation of oncology clinical pharmacy practice in China, and the aim of the study. The questionnaire consists of 55 questions, which was structured to mainly cover five aspects: 1 ) the basic information of the participating pharmacists; 2) the basic working status of the pharmacists; 3 ) the contents and features of oncology clinical pharmacy services; 4) the pharmacists' impression on the perceptions of other healthcare professionals; 5) the self-evaluation of the pharmacists.

The Participants And Methods 
The survey was conducted in January 2019. The participants of the survey were clinical pharmacists serving cancer-related departments from hospitals with different tiers, from different provinces and municipalities. The pharmacists answered questions on the WeChat platform through online links.

Data analysis

A total of 167 questionnaires were recycled, of which one had obvious unreasonable data, therefore 166 questionnaires were considered valid and remained for data analysis. Data were analyzed with descriptive statistics.

\section{Results}

The pharmacists' characteristics

The respondents were from 29 provinces (or autonomous regions or municipalities) in China. Among them, Hunan $(12.65 \%, \mathrm{n}=21)$ and Beijing $(12.05 \%, \mathrm{n}=20)$ were the provinces and cities with the largest number of clinical pharmacists participating in the survey, followed by Qinghai $(11.45 \%, n=19)$, Jilin $(7.83 \%, n=13)$, and Anhui $(6.02 \%, n=10)$. The vast majority of the pharmacists came from Tier III hospitals $(92.17 \%, n=153)$, with Tier II and Tier I hospitals accounting for $7.83 \%(n=13)$ and $0 \%(n=0)$, respectively. Respondents from general hospitals and specialized hospitals accounted for $48.8 \%(n=81)$ and $51.2 \%(n=85)$, respectively. The average number of clinical pharmacists in the hospital was 12.06 . There were $56.63 \%$ of the pharmacists obtained a Master's degree $(n=94)$, and $51.2 \%$ of the pharmacists had intermediate professional titles $(n=85)$. Most of the training duration for clinical pharmacists was one year $(49.66 \%, n=72)$. The average working experience for clinical pharmacy was 4.86 years. There were $65.66 \%$ of the respondents serving oncology internal medicine departments $(n=109)$, followed by the integration departments of both oncology surgery and internal medicine $(20.48 \%, n=34)$. The average working hours for the pharmacists to serve clinical departments was 3.27 hours per working day. (details seen in Table 1) 
The characteristics and the general service situation of the clinical pharmacists

1. Regions of the pharmacists
Hunan $(12.65 \%, \mathrm{n}=21) \quad$ Zhejiang $(2.41 \%, \mathrm{n}=4)$

Beijing $(12.05 \%, n=20) \quad$ Heilongjiang $(1.81 \%, n$

Qinghai $(11.45 \%, n=$

19)

Jilin $(7.83 \%, n=13)$

Anhui $(6.02 \%, \mathrm{n}=10)$

Shanghai $(5.42 \%, n=9)$

Jiangsu $(4.82 \%, n=8)$

Fujian $(4.82 \%, n=8)$

Guangxi $(4.82 \%, n=8)$

Sichuan $(4.22 \%, n=7)$

Hubei $(3.61 \%, n=6)$

Inner Mongolia (2.41\%,

$\mathrm{n}=4$ )

Henan $(2.41 \%, n=4)$

Gansu $(2.41 \%, n=4)$

Shandong $(2.41 \%, n=$

4)
=3)

Guangdong $(1.20 \%, n=$

2)

Xinjiang $(1.20 \%, n=2)$

Yunnan $(0.6 \%, n=1)$

Tianjin $(0.6 \%, n=1)$

Shanxi [shăn xi] $(0.6 \%$, $n=1$ )

Shanxi [shān xĩ] $(0.6 \%$, $n=1$ )

Chongqing $(0.6 \%, n=1)$

Guizhou $(0.6 \%, n=1)$

Hebei $(0.6 \%, n=1)$

Ningxia $(0.6 \%, n=1)$

Jiangxi $(0.6 \%, n=1)$

Liaoning $(0.6 \%, n=1)$

2. The level of the pharmacists' hospitals

Tier III hospitals $(92.17 \%, n=153)$

Tier II hospitals $(7.83 \%, n=13)$

Tier I hospitals $(0 \%, \mathrm{n}=0)$

3. Specialty coverage of the pharmacists' hospitals

4. Academic degree of the pharmacists
General hospitals $(48.8 \%, \mathrm{n}=81)$

Specialized hospitals $(51.2 \%, \mathrm{n}=85)$

Doctor's degree $(7.83 \%, \mathrm{n}=13)$

Master's degree $(56.63 \%, \mathrm{n}=94)$

Bachelor's degree $(33.13 \%, \mathrm{n}=55)$

College's degree $(2.41 \%, \mathrm{n}=4)$

Others $(0 \%, \mathrm{n}=0)$

5. The professional titles of the pharmacists

Chief pharmacist $(4.82 \%, \mathrm{n}=8)$

Associate chief pharmacist $(16.27 \%, \mathrm{n}=27)$

Pharmacist-in-charge $(51.2 \%, \mathrm{n}=85)$

Junior pharmacist $(27.71 \%, \mathrm{n}=46)$

Others $(0 \%, \mathrm{n}=0)$

6. The average number of clinical pharmacists $\quad 12.06$ in the hospital

7. The average working experience for clinical $\quad 4.86$ years pharmacy (year)

8. The distribution of full-time and part-time clinical pharmacists
Full time $(78.31 \%, \mathrm{n}=130)$

Part time $(21.69 \%, \mathrm{n}=36)$ 
9. The training duration for clinical pharmacists
One year $(49.66 \%, \mathrm{n}=72)$

More than one year $(22.76 \%, \mathrm{n}=33)$

Less than half of a year $(15.17 \%, n=22)$

Half of a year $(12.41 \%, n=18)$

Mainly oncology internal medicine departments

$(65.66 \%, \mathrm{n}=109)$

Integration of both oncology surgery and internal

medicine departments $(20.48 \%, \mathrm{n}=34)$

Others $(8.43 \%, \mathrm{n}=14)$

Mainly oncology surgery departments $(5.42 \%, n=$ 9)

11. The specialty of the clinical departments that pharmacists served
General oncology $(43.98 \%, n=73)$

Oncology with a specific tumor type $(37.95 \%, n=$ 63)

Infectious Diseases $(5.42 \%, \mathrm{n}=9)$

ICU $(1.81 \%, \mathrm{n}=3)$

Others $(10.84 \%, \mathrm{n}=18)$

12. The average working hours for the pharmacists to serve in clinical departments

3.27 hours per working day

The Current Status Of Oncology Clinical Pharmacy Services

According to the general coverage of clinical pharmacy services in China, commonly practiced services were investigated, such as order verification, drug counselling, pharmacy consultation, multidisciplinary team (MDT) rounding, in-service education and other service items. The details of them were as follows:

Concerning order verification, there were $68.07 \%(n=113)$ of the pharmacists responded that their hospital had not established pre-audit order verification (or prescription verification), but $58.43 \%$ of the respondents' hospital information systems had established order sets $(n=97)$. In most cases $(30.12 \%, n$ $=50$ ), order verification accounted for $20-39 \%$ of the working hours per day for clinical pharmacists. The average number of patients for order verification per day per pharmacist was 48.6 . There were $44.54 \%$ (n $=53$ ) of the pharmacist responded that the number of orders verified per day per pharmacist was less than 100. The top five types of irrational orders identified by pharmacists were (multiple choices) irrational dose or frequency $(70.59 \%, n=84)$, irrational vehicle $(56.3 \%, n=67)$, irrational drug selection $(38.66 \%, n=46)$, irrational duration of treatment $(29.41 \%, n=35)$ and adverse drug-drug interactions $(15.97 \%, n=19)$. In most cases $(75.64 \%, n=90)$, the acceptance rate of pharmacists' intervention was over $50 \%$. The average daily number of patients monitored by the clinical pharmacists (with pharmacotherapy records) was 3.32. (as seen in Fig. 1)

In terms of drug counselling, the top five drug categories counselled were (multiple choices): antimicrobials $(73.49 \%, n=122)$, analgesics $(37.95 \%, n=63)$, chemotherapy drugs $(33.73 \%, n=56)$, antiemetics $(14.46 \%, n=24)$ and nutritional support drugs $(11.45 \%, n=19)$. The top five most frequently counselled questions were about (multiple choices): drug selection $(63.86 \%, n=106)$, dosage and frequency $(51.2 \%, n=85)$, management of adverse reactions $(50.6 \%, n=84)$, dose adjustment $(48.19 \%, n$ $=80$ ) and drug preparations $(13.86 \%, n=23)$. (as seen in Fig. 2 ) 
The coverage of other clinical pharmacy services and the workload of each service item were summarized in Table 2. 
Table 2

The coverage of other clinical pharmacy services and pharmacists' workload

No. Service items

The average number of pharmacy consultation per month per pharmacist

2 The number of in-service education provided for clinical departments about rational drug use (per pharmacist per year)
Workload of pharmacists (rank from high to low)

$1-5(43.37 \%, n=72)$

Zero $(34.94 \%, \mathrm{n}=58)$

$6-10(9.64 \%, n=16)$

$11-15(6.02 \%, \mathrm{n}=10)$

ه15 $(6.02 \%, n=10)$

$1-5(67.47 \%, \mathrm{n}=112)$

Zero $(20.48 \%, \mathrm{n}=34)$

$6-10(6.02 \%, n=10)$

ه15 $(4.22 \%, \mathrm{n}=7)$

$11-15(1.81 \%, n=3)$

3 The average number of audiences for every inservice education

$10-20(49.24 \%, \mathrm{n}=65)$

$\nabla 10(24.24 \%, \mathrm{n}=32)$

$21-50(17.42 \%, \mathrm{n}=23)$

$\nabla 50(9.09 \%, n=12)$

$4 \quad$ The number of patients receiving medication reconciliation service performed by pharmacists (per pharmacist per day)

$1-5(53.61 \%, n=89)$

Zero $(41.57 \%, \mathrm{n}=69)$

$6-10(3.61 \%, n=6)$

$\nabla 10(1.2 \%, n=2)$

2.49 per month

5 The average number of MDTs held in the clinical departments that the pharmacists served

6 The frequency of pharmacists participating in MDT

Occasional $(35.54 \%, \mathrm{n}=59)$

Frequently $(31.93 \%, \mathrm{n}=53)$

Never $(24.7 \%, \mathrm{n}=41)$

Always $(7.83 \%, \mathrm{n}=13)$

$7 \quad$ The number of patients receiving patient education service performed by pharmacists (per pharmacist per day)

$1-5(71.69 \%, n=119)$

Zero $(12.65 \%, \mathrm{n}=21)$

$6-10(10.24 \%, n=17)$

$\mathrm{\nabla 10}(5.42 \%, \mathrm{n}=9)$

$1-5(70.48 \%, n=117)$

Zero $(13.86 \%, \mathrm{n}=23)$

$6-10(8.43 \%, n=14)$

$11-15(4.82 \%, \mathrm{n}=8)$

$\nabla 15(2.41 \%, n=4)$

9 The number of patients receiving therapeutic drug monitoring service performed by pharmacists (per pharmacist per month)
Zero $(71.08 \%, \mathrm{n}=118)$

$1-5(22.29 \%, n=37)$

ه20 $(3.61 \%, n=6)$

6-10 $(3.01 \%, n=5)$

$11-20(0 \%, n=0)$ 


\section{No. Service items}

10 The number of patients that received pharmacogenetic/pharmacogenomic services performed by pharmacists (per pharmacist per month)
Workload of pharmacists (rank from high to low)

Zero $(78.92 \%, \mathrm{n}=131)$

$1-5(16.27 \%, n=27)$

6-10 $(3.01 \%, n=5)$

ه20 $(1.2 \%, \mathrm{n}=2)$

$11-20(0.6 \%, n=1)$

11 Whether pharmacotherapy guidelines or protocols was established in hospital

No $(55.42 \%, \mathrm{n}=92)$

Yes $(44.58 \%, n=74)$

12 The frequency of pharmacists participating in establishing pharmacotherapy guidelines (protocols)

Occasionally $(41.89 \%, \mathrm{n}=31)$

Frequently $(31.08 \%, \mathrm{n}=23)$

Hardly $(27.03 \%, n=20)$

PS: The effective responses for this question was 74

$1-3(62.05 \%, \mathrm{n}=103)$

Zero $(27.11 \%, \mathrm{n}=45)$

$4-6(4.22 \%, \mathrm{n}=7)$

$\bigotimes 10(4.22 \%, \mathrm{n}=7)$

$7-10(2.41 \%, n=4)$
14 The extent of viewability of pharmacotherapy records wrote by pharmacists

Not viewable in hospital information system $(54.55 \%, n=66)$

Only viewable to the pharmacists

themselves $(33.06 \%, n=40)$

Only viewable to authorized

professionals in the hospital $(9.92 \%, \mathrm{n}$

$=12$ )

Viewable to all the professionals in the hospital $(2.48 \%, \mathrm{n}=3)$

PS: The effective responses for this question was 121

15 The ways for recording or calculating the workload for clinical pharmacy services

By handwriting $(33.73 \%, \mathrm{n}=56)$

By self-created electronical tables (e.g.
EXCEL) $(30.72 \%, n=51)$

No formal recording $(27.11 \%, \mathrm{n}=45)$

By hospital information system

$(8.43 \%, n=14)$

Less than $30 \%(63.86 \%, \mathrm{n}=106)$

$60-99 \%(14.46 \%, \mathrm{n}=24)$

$30-59 \%(14.46 \%, n=24)$

$100 \%(7.23 \%, \mathrm{n}=12)$

17 The number of pharmacists' participations in outpatient drug counselling (or pharmacist clinic) (per pharmacist per month)
Zero $(42.77 \%, \mathrm{n}=71)$

$1-2$ times $(32.53 \%, \mathrm{n}=54)$

$3-4$ times $(16.27 \%, \mathrm{n}=27)$

$5-8$ times $(4.82 \%, \mathrm{n}=8)$

$\llbracket 8$ times $(3.61 \%, n=6)$

PS: usually pharmacists serving in outpatient drug counselling for half a day was counted as "one time" 


\section{No. Service items}

8 The number of "patient visits" in outpatient drug counselling (or pharmacist clinic) (per pharmacist per one-time service)

19 The frequency of pharmacists participating in improving the pharmacotherapy procedures in the hospital information system

20 Whether pharmacists participated in community contracting services with patients

21 The number of current pharmacy research projects (per pharmacist)
Workload of pharmacists (rank from

high to low)

$1-10(78.92 \%, \mathrm{n}=131)$

$11-20(15.06 \%, \mathrm{n}=25)$

$21-30(3.01 \%, n=5)$

$\varangle 30(3.01 \%, n=5)$

Occasionally $(45.78 \%, \mathrm{n}=76)$

Frequently $(33.13 \%, \mathrm{n}=55)$

Hardly $(21.08 \%, n=35)$

No $(97.59 \%, n=162)$

Yes $(2.41 \%, n=4)$

$1-2(50 \%, n=83)$

Zero $(43.37 \%, \mathrm{n}=72)$

$3-4(4.22 \%, \mathrm{n}=7)$

$\nabla 4(2.41 \%, n=4)$

The pharmacists' impression on the perceptions of other healthcare professionals

According to the results, more than half of the pharmacists responded that other healthcare professionals addressed them as "teacher" $(51.81 \%, \mathrm{n}=86)$, and only $28.31 \%$ of them responded that other healthcare professionals addressed them as "pharmacist" $(n=47)$. Concerning how common that other healthcare professionals knew the nature of pharmacists' work, $57.23 \%(n=95)$ of the pharmacists responded "some of the healthcare professionals knew the nature of my work" and $24.1 \%(n=40)$ of the pharmacists responded "most of the healthcare professionals knew the nature of my work". Pharmacists believed that among the pharmaceutical services they provided to the clinical departments, the five most popular service categories were (multiple choices): drug counselling $(69.28 \%, n=115)$, patient education $(61.45 \%, n=102)$, in-service education $(42.77 \%, n=71)$, reporting and management of adverse drug reactions $(35.54 \%, n=59)$, and order verification (or prescription verification) $(29.52 \%, n=49)$ (seen in Fig. 3).

Self-evaluation Of The Pharmacists

In terms of self-evaluation of the pharmacists, the top five pharmacotherapy problems that they were not good at were (multiple choices): anticoagulation management $(67.47 \%, \mathrm{n}=112)$, parenteral and enteral nutrition $(61.45 \%, \mathrm{n}=102)$, hypertension and diabetes management $(51.81 \%, \mathrm{n}=86)$, selection of chemotherapy regimens and dose adjustments $(33.73 \%, n=56)$, and anti-infective treatment $(28.31 \%, n=$ 47). Pharmacists believed that among the subjects that they were relatively not good at but very necessary, the top five ones were pharmaceutical research $(48.8 \%, n=81)$, pathology $(46.99 \%, n=78)$, 
pharmacokinetics and pharmacodynamics $(40.96 \%, n=68)$, pharmacogenomics $(40.96 \%, n=68)$, oncology internal medicine $(37.95 \%, \mathrm{n}=63)$. With regard to the relationship between pharmacists and other healthcare professionals, $48.8 \%(n=81)$ of the pharmacists responded "very harmonious" and $46.39 \%$ of them responded "in general harmonious" $(n=77)$. When being asked about how do they perceive their specialized services in clinical departments, $56.63 \%$ of the pharmacists responded "positive addition" ( $n=94), 13.86 \%$ of them responded "indispensable" $(n=23)$, and $29.52 \%$ of them responded "insignificant" ( $n=49)$.

Among the categories of clinical pharmacy services, the top five services that were considered by pharmacists to be inadequately performed were the establishment of pharmacotherapy protocols or guidelines $(51.2 \%, n=85)$, therapeutic drug monitoring $(48.19 \%, n=80)$, pharmacy consultation $(44.58 \%$, $n=74)$, pharmacogenomics services $(43.98 \%, n=73)$ and medication reconciliation $(37.95 \%, n=63)$. Additionally, insufficiency of professional competency $(36.75 \%, n=61)$ ranked as the most frequent answer for "the biggest barrier for career development of pharmacists", followed by perception of the hospital leaders on pharmacy department $(22.29 \%, n=37)$, and national or institutional policies $(21.69 \%$, $n=36$ ). In terms of salary and occupational well-being, most pharmacists thought that their salary was relatively low (compared to their competency) $(58.43 \%, n=97)$. The average score for occupational wellbeing was 6.27 out of 10 points. (seen in Fig. 4 and Fig. 5)

\section{Discussion}

Basic clinical services provided by oncology pharmacists in China are relatively comprehensive, but highlevel clinical services need to be expanded

From the survey results, the clinical services currently covered by oncology pharmacists mainly included order verification, drug counselling, pharmacy consultation, in-service education, medication reconciliation, participation in multidisciplinary rounding, patient education, reporting and management of adverse drug reactions, therapeutic drug monitoring (TDM), pharmacogenetic/pharmacogenomic services, participating in outpatient drug counselling, etc., and they were basically the similar services as those in Europe, America, Japan, and other countries whose clinical pharmacy is relatively welldeveloped. However, some advanced services, such as establishment of pharmacotherapy guidelines (or protocols), therapeutic drug monitoring, pharmacy consultation, pharmacogenetic/pharmacogenomic services need to be expanded.

At present, the work of therapeutic drug monitoring (TDM) in China is developing rapidly and related standards have been recently established [7]. Division of Therapeutic Drug Monitoring, Chinese Pharmacological Society conducted a nationwide survey about the current status of TDM work in China. According to the 2017 data (credit to Division of Therapeutic Drug Monitoring, Chinese Pharmacological Society, data not published), altogether there were 203 hospitals implemented TDM. The drugs monitored included 16 types of antibacterial drugs, 8 types of immunosuppressive drugs, 3 types of theophylline drugs, 5 types of anti-tuberculous drugs, 8 types of antitumor drugs, 22 types of antidepressants, 6 types 
of antiepileptic drugs, 4 types of anti-viral drugs, 2 types of antiarrhythmic drugs, 1 type of anticoagulant drugs, 4 types of poisonous pesticides, and 7 types of other drugs, in total 86 types. In the year of 2017, 1032452 samples were tested and 203062 reports were interpreted by pharmacists.

In the field of oncology, the development of therapeutic drug monitoring is relatively slow, mainly due to the controversy over the target range of anti-tumor drugs and the slow development of detection technology. In addition, the combination of anti-tumor drugs often makes it difficult to study the individual pharmacokinetics of individual drugs. The above may be part of the reasons for the insufficiency participation of pharmacists in oncology TDM. However, the therapeutic window of antitumor drugs is usually narrow and the pharmacokinetics of them are highly diversified, therefore oncology TDM still has great development potential [8]. In China the commonly monitored chemotherapy drugs are methotrexate, fluorouracil, paclitaxel, etoposide, irinotecan, mitotan, cyclophosphamide, platinum, etc.; and the commonly monitored targeted drugs are imatinib, bevacizumab, rituximab, trastuzumab, etc. In the current survey, the majority of oncology pharmacists $(71.08 \%, n=118)$ did not participate in TDM. For the small number of pharmacists who participated in this work, the number of patients that they monitored were within the range of 1-5 per month per pharmacist, indicating that there is great potential for oncology TDM to be further advanced.

According to this survey, most of the pharmacists were not involved in $(78.92 \%)$ pharmacogenetics or pharmacogenomics services. In fact, even in the United States (US), where clinical pharmacy is relatively well-developed, pharmacogenetics or pharmacogenomics services have not widely been implemented in clinical practice. A descriptive literature review was conducted on the current status of the integration of pharmacogenomics into clinical pharmacy practice in US institutions, and found that different hospitals have incorporated pharmacogenomics/precision medicine into different service models. Pharmacists played important roles in these services. The examples of the service models were molecular tumor board, pharmacogenomics (PGx)-based medication therapy management, PGx-based anticoagulation or antiplatelet outpatient services, etc., and the main roles of pharmacists in these services were to screen target patients, provide doctors with testing advices, interpret test results, assist with adjustment for treatment plans, educate patients, etc [9]. Clinical pharmacists in China can learn from US institutions to incorporate pharmacogenetics/pharmacogenomics/precision medicine into clinical pharmacy practices and actively expand their roles. Additionally, this work also requires strong support from National Health Commission of the People's Republic of China, National Healthcare Security Administration and hospital departments.

The working time that oncology pharmacists spend on clinical services need to be increased, and the evaluation system for clinical pharmacy performance need to be improved

In terms of working hours, the results of this survey indicated that the average working time of all the clinical pharmacists (part-time + full-time) dedicating on clinical services was 3.27 hours per working day. Among the 130 full-time participating pharmacists, the average working time for clinical services was 3.3 hours, which was close to the overall average value. The results showed that most oncology clinical 
pharmacists, whether part-time or full-time, whose at least half of their daily working time was occupied by non-clinical services. A survey conducted in 56 Tier III hospitals from 17 provinces (autonomous regions or municipalities) across the country, showed similar results as those in our survey: when pharmacists were asked about the proportion of time spent in clinical services, $23.46 \%$ of them responded more than $30 \%, 35.55 \%$ of the pharmacists responded more than $50 \%$, and only $7.65 \%$ of them responded more than $90 \%, 13.19 \%$ of them even responded no participation in clinical services [10]. According to the requirement of "Detailed Implementation Rules for the Evaluation Standards for Tier III Hospitals (2011 Edition)", clinical pharmacists should spend more than $85 \%$ of their daily working hours to participate in clinical-related services [11]. Both of the surveys showed that the time pharmacists spent on clinical pharmacy services was insufficient. In US, full-time clinical pharmacists spend almost all of their working time on clinical services. In addition, in this survey, when asked about the weight of clinical service performance in the overall performance assessment, a considerable number of the pharmacists $(63.86 \%, n=106)$ reported less than $30 \%$, and the proportion of pharmacists reported $30-59 \%$ and $60-$ $99 \%$ were the same, both being $14.46 \%$ ( $n=24$ for both groups). In the future, with sufficient staffing, it should be considered to allow clinical pharmacists to be freed from non-clinical work as much as possible to focus mainly on serving in clinical departments, so as to promote the in-depth and sustainable development of clinical pharmacy in China.

The future directions for continuing education and training of oncology pharmacists

With regard to self-assessment of the oncology pharmacists, anticoagulation management, nutritional support, hypertension and diabetes management, chemotherapy regimen and dose adjustment, and antiinfective treatment ranked at top 5 expertise that pharmacists thought they were not good at. This may be due to the training content of oncology pharmacists. The training of oncology clinical pharmacists in China is mainly about learning chemotherapy regimens for different tumor types, and there is no systematic training in internal medicine or general pharmacotherapy. However, chemotherapy or surgeryinduced thrombosis, management of comorbidities, tumor-related nutrition and infection etc. are very common in clinical practice, therefore oncology pharmacists may feel the insufficiency of expertise when providing clinical services. When asked about "what are the disciplines that you feel not good at but very necessary", most pharmacists responded pharmaceutical research, pathology, pharmacokinetics and pharmacodynamics, pharmacogenomics, and oncology internal medicine. It is very interesting that scientific research ranked at first for this question. Nowadays in most hospitals in China, no matter the position is clinical or scientific, performance on scientific research weighted a lot in the overall performance for healthcare professionals, which also explains to some extent the reasons that clinical pharmacists could not spend most of their working time on clinical services. In the future when considering continuing education of oncology clinical pharmacists, the training of the abovementioned disciplines and service types should be strengthened, in order to meet the need of self-improvement for oncology pharmacists, and promote the healthy and sustainable development of clinical pharmacy teams. 
Multiple measures need to be taken to improve the professional identity and overall well-being of oncology pharmacists

According to the results of this survey, most of the pharmacists were addressed as "teacher" rather than "pharmacist" by other healthcare professionals, indicating the professional identity of pharmacists were not very clear. However, it is worth noting that most of the pharmacists thought that other healthcare professionals understood their job nature, and most of the pharmacists responded "very harmonious" concerning the relationship between pharmacists and other healthcare professionals. Additionally, most of pharmacists perceived their specialized services in clinical departments as a "positive addition" $(56.63 \%, n=94)$ or even "indispensable" $(13.86 \%, n=23)$, indicating the roles of pharmacists were increasingly acknowledged by peers. On the contrary, there were almost $30 \%$ of the pharmacists considering their work "insignificant" to clinical departments, indicating that some pharmacists did not have a strong sense of self-identity. Similarly, a previous survey on clinical pharmacists in more than 30 hospitals in Beijing showed that $33.3 \%$ of the clinical pharmacists (150/450) were proud of their profession; $42.0 \%$ (189/450) of those felt general pride; $13.6 \%$ of them felt little pride $(61 / 450)$ and $11.1 \%$ of them (50/450) felt no pride or consider their work unimportant [12]. In general, a pharmacist must first have a sense of pride for their own profession, and then he will voluntarily improve his professional expertise and communicate with other healthcare professionals with confidence. Therefore, concerning future training and continuing education programs, it is suggested to appropriately strengthen selfidentity training and share more excellent service cases conducted by pharmacists with the pharmacy trainees.

When asked about whether it was matching between their professional expertise and salary, most pharmacists $(58.43 \%)$ thought their salary was relatively low, and the comprehensive score for professional wellbeing was only 6.27 out of 10 points. According to several pieces of previous research, there are three domains for job satisfaction scale: payment-promotion, management-interpersonal relationship, and organization-communication [13]. Workload, stress, advancement opportunities, job security, autonomy, fairness in the workplace, relationship with supervisors and colleagues, work flexibility, and working atmosphere are factors contributing to pharmacists' job satisfaction [14]. There are generally two ways to improve professional happiness (job satisfaction) for oncology clinical pharmacists: at the intrinsic level, it is pharmacists' responsibility to work harder and smarter to improve their professional competency, strengthen collaboration with other healthcare professionals, establish more guidelines or consensuses, conduct more science communication activities and gather more data to prove their values to hospital management leaders; at the extrinsic level, it is suggested that central and local government, all sectors of society, and hospital management departments pay more attention and offer more support to the discipline of clinical pharmacy, so as to work together to promote greater confidence and strength for pharmacists.

\section{Limitations Of The Study}


There are altogether 34 provincial-level administrative regions in China. However, the participants in the survey were only from 29 of them. Thus, the current situation that described and discussed in this article can only reflect the fact in most parts of China.

\section{Conclusion}

The value of oncology clinical pharmacists is being increasingly recognized and the services that they provide are relative comprehensive, but some advanced services, such as TDM, pharmacy consultation, and pharmacogenetic/pharmacogenomic services need further expansion. Increasing the time serving in clinical units, optimizing performance evaluation and strengthening professional competency are possible ways for pharmacists to provide superior services in the future.

This study showed the current service of oncology clinical pharmacists in most parts of China, which may provide reference for policy makers, promote international communications and shed light on future development of oncology clinical pharmacy in China.

\section{Declarations}

\section{Ethics approval and consent to participate}

This study does not need formal ethic approval and this complies with "Ethical Review Methods for Biomedical Research Involving Humans", in which the activities that need ethic approval do not include this kind of survey [15].

Concerning the informed consent, in our questionnaire we have mentioned that "the significance of the survey was to collect information of the current status of oncology clinical pharmacy services, so as to identify related problems and facilitate its further development, and we acknowledged the pharmacists who participated in this study of making such contributions." This could be considered as the written consent and the subjects knew that we would collect and analyze these data. Additionally, we did not collect any information about the identity of the pharmacists (e.g. name, gender)

\section{Consent for publication}

Not applicable.

\section{Availability of data and materials}

The datasets during and/or analyzed during the current study are available from the corresponding author on reasonable request.

\section{Competing interests}

The authors declare that they have no competing interests. 


\section{Funding}

This work was supported by National Natural Science Foundation of China (Grant number 81803511) and The Chinese Academy of Medical Sciences Innovation Funds for Medical Sciences (CIFMS, Grant nos. 2016-I2M-1-001, 2017-I2M-2-003, and 2017-I2M-1-005). The funding sources supported data storage for this study.

\section{Authors' contributions}

YTW is responsible for the design and execution of the survey and the writing of the article. YYD, JY and $\mathrm{HYZ}$ offer important suggestions of the questionnaire design. $\mathrm{ZC}$ revised the manuscript. GHL is responsible for the design and supervision of the study and is the corresponding author.

\section{Acknowledgements}

We thank all the pharmacists who participated in this study.

\section{Authors' information}

The corresponding author, GHL, is a professor of Hospital Pharmacy, and the Director of Department of Pharmacy, National Cancer Center of China. She is also the President of Oncology Clinical Pharmacy Committee of China Anti-Cancer Association. The members of the committee (over 100 people) are from different parts of China, and most of them are directors of pharmacy departments from Chinese cancerspecialized hospitals. The current survey was conducted with the guidance and supervision of the committee and the results of the study will be references for future development of oncology clinical pharmacy.

YYD is the Vice Director of Department of Pharmacy, National Cancer Center of China. She is also the Secretary General, Oncology Clinical Pharmacy Committee of China Anti-Cancer Association.

YTW, JY, HYZ and ZC are oncology clinical pharmacists serving in different internal and surgical oncology departments of National Cancer Center of China.

\section{List Of Abbreviations}

Multidisciplinary team (MDT)

Therapeutic drug monitoring (TDM)

United States (US)

Pharmacogenomics (PGx)

Chinese Academy of Medical Sciences Innovation Funds for Medical Sciences (CIFMS) 


\section{References}

1 Allemani C, Matsuda T, Di Carlo V, Harewood R, Matz M, Niksic M, et al. Global surveillance of trends in cancer survival 2000-14 (CONCORD-3): analysis of individual records for 37513025 patients diagnosed with one of 18 cancers from 322 population-based registries in 71 countries. Lancet 2018: 391: 1023-1075.

2 National Health Commission of the People's Republic of China. Notice on Strengthening the Management of Standardized Cancer Diagnosis and Treatment. Bulletin of the National Health and Family Planning Commission of the People's Republic of China 2016: 03: 3-5.

3 National Health Commission of the People's Republic of China. Guidelines for clinical application of new anti-tumor drugs. Journal of Multidisciplinary Cancer Management (Electronic Version) 2019: 5: $35-54$.

$4 \quad$ Li GH, Yang J, Dai Z, Dai YY, Wang C, Dong M, et al. Expert consensus on prescription review of anti-tumor medications--Lung cancer. Chinese Pharmaceutical Journal 2019: 54: 847-854.

5 Li GH, Hao ZY, Chen Z, Chai LM, Zhou HY, Liu GX, et al. Expert consensus on prescription review of anti-tumor medications--Liver cancer. Chinese Pharmaceutical Journal 2019: 54: 539-1542.

6 Li GH, Dong M, Chen Z, Wu DY, Dai YY, Liu J, et al. Expert consensus on prescription review of anti-tumor medications--Colorectal cancer. Chinese Pharmaceutical Journal 2019: 54: 1361-1366.

7 Zhang XL, Miao LY, Chen WQ. The Expert Consensus on the Standards of Therapeutic Drug Monitoring (2019 Edition). Evaluation and Analysis of Drug-use in Hospitals of China 2019: 19: 897-898.

8 Zhang HY, Dai Z. Therapeutic drug monitoring of anticancer drugs: research advances. Journal of International Pharmaceutical Research 2018: 45: 333-338.

9 Wang YT, Merl MY, Yang J, Zhu ZX, Li GH. Opportunities for pharmacists to integrate pharmacogenomics into clinical practice. The Pharmacogenomics Journal 2019, DOI: 10.1038/s41397019-0119-8. [Epub ahead of print].

10 Li J, Zhang CZ, Chen QX, Yang JD, Long R, Meng L et al. Investigation on the demands and evaluation of medical personnel and patients towards clinical pharmacists in 56 Tier III Class A hospitals of 17 provinces (regions, cities). China Pharmacy 2018: 29: 1136-1139.

11 Ministry of Health of People's republic of China. Notice of the General Office of the Ministry of Health on Printing and Distributing the "Implementation Rules for the Evaluation Standards for Tertiary Hospitals (2011 Edition)"2011: 45.

12 Yang H, Zhao LH, Guo T, Feng X. Investigation and analysis of medical ethics education for clinical pharmacists. China Medicine 2014: 9: 1704-1706. 
13 lorga M, Dondas C, Soponaru C, Antofie I. Determinants of Hospital Pharmacists' Job Satisfaction in Romanian Hospitals. Pharmacy (Basel) 2017: 5. pii: E66. DOI:

10.3390/pharmacy5040066.

14 Carvajal MJ, Popovici I. Gender, age, and pharmacists' job satisfaction. Pharmacy Practice (Granada) 2018: 16: 1396.

15 National Health and Family Planning Commission of the People's Republic of China. Human Ethics Review Methods for Biomedical Research 2016.

http://www.gov.cn/gongbao/content/2017/content_5227817.htm. Accessed 31 Jan. 2020.

\section{Figures}

a

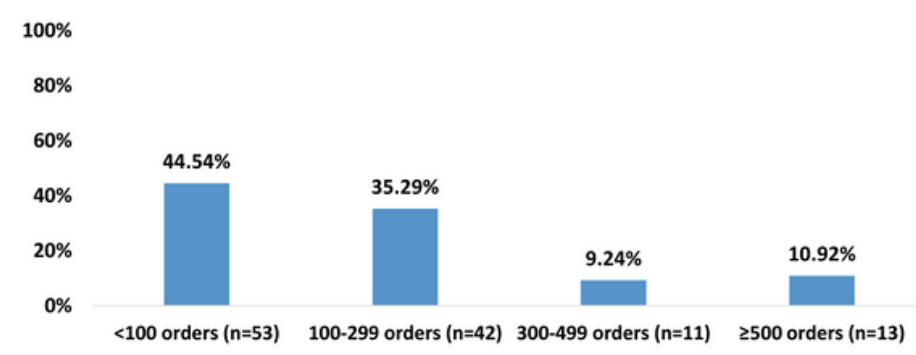

$\mathrm{c}$

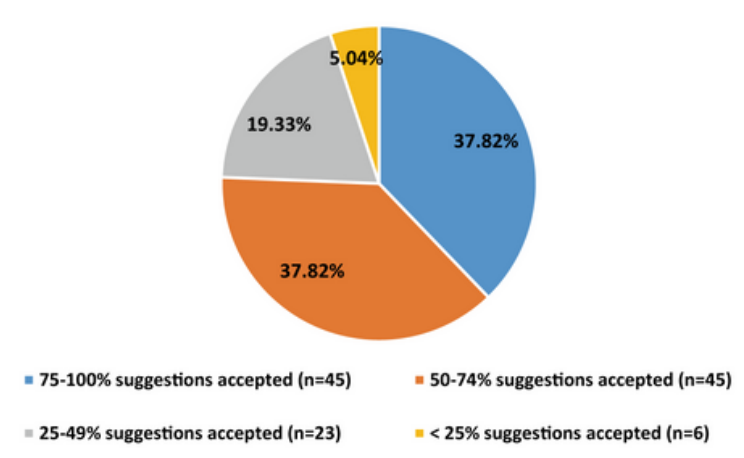

b

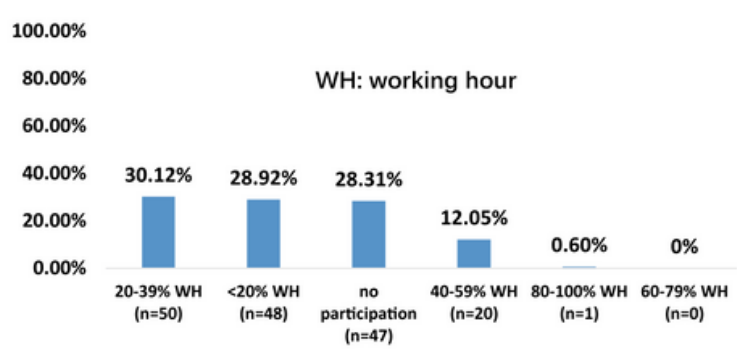

$\mathrm{d}$

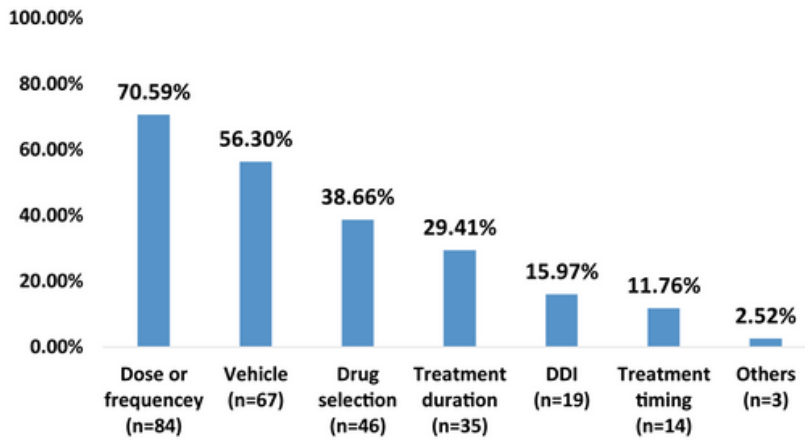

\section{Figure 1}

Details for the current status of order (prescription verification). a. the number of orders verified per day per pharmacist; $b$. the ratio of the time spent on order verification to the whole working hour; $c$. the acceptance rate of pharmacists' suggestions during order verification; $d$. types of problematic orders identified by pharmacists (answers with multiple choices) 


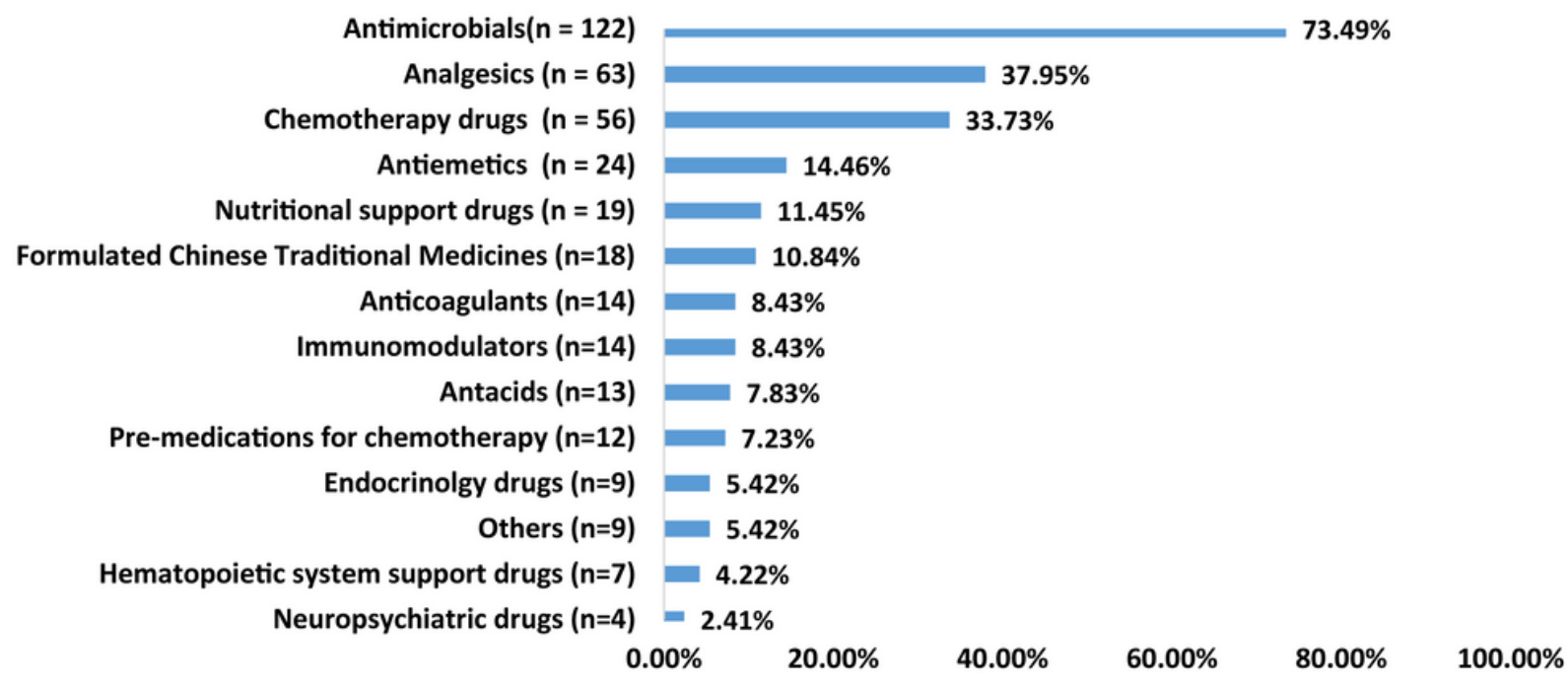

b

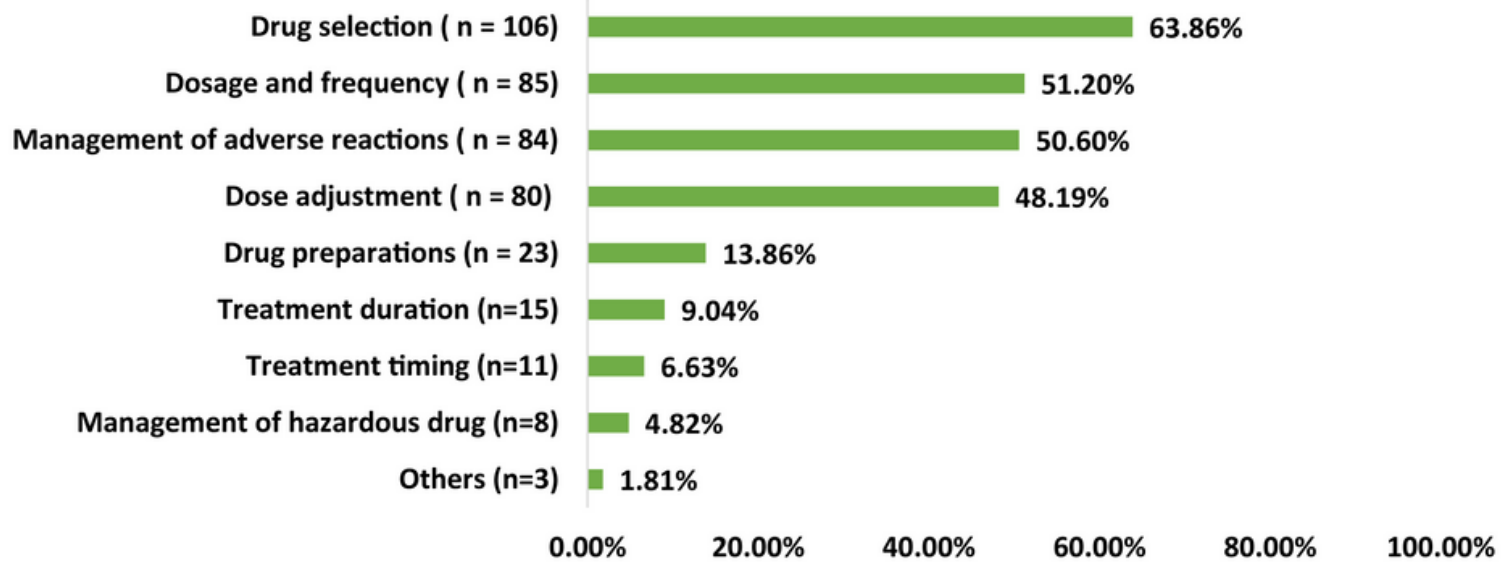

\section{Figure 2}

Details for the current status of drug counselling. a. the drug categories that were frequently asked in drug counselling (multiple choices); b. the question types that were frequently asked in drug counselling (multiple choices) 
a

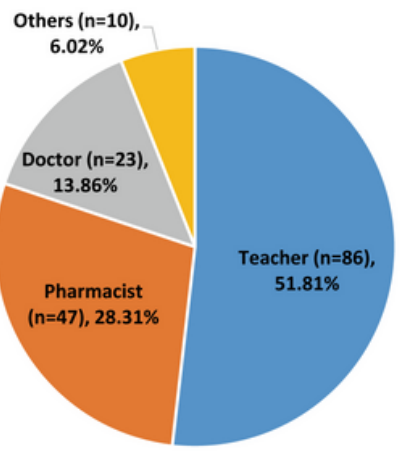

$\mathrm{b}$

Some of the healthcare professionals know ( $n=95)$

Most of the healthcare professionals know $(n=40)$

Only a small part of healthcare professionals know $(n=24)$

Almost none of them know $(n=7) \quad 4.22 \%$

$\mathrm{c}$

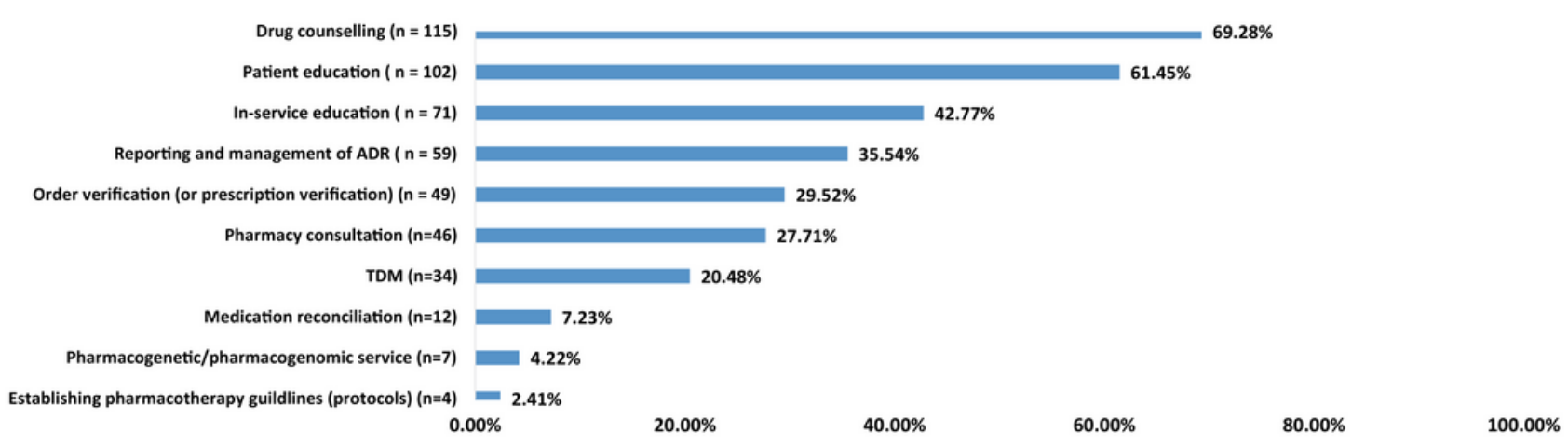

\section{Figure 3}

The pharmacists' impression on the perceptions of other healthcare professionals. a. the way that other healthcare professionals addressed pharmacists; $b$. how common that other healthcare professionals knew the nature of pharmacists' work. c. the service types that were welcomed by clinical departments (multiple choices) 


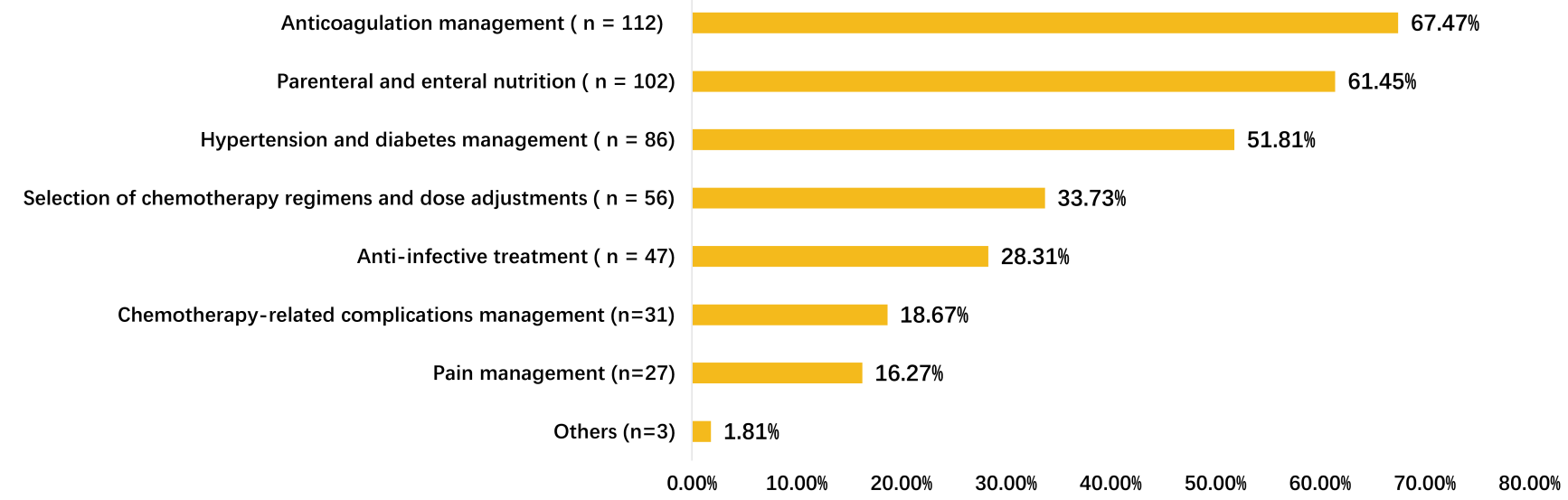

$\mathrm{b}$

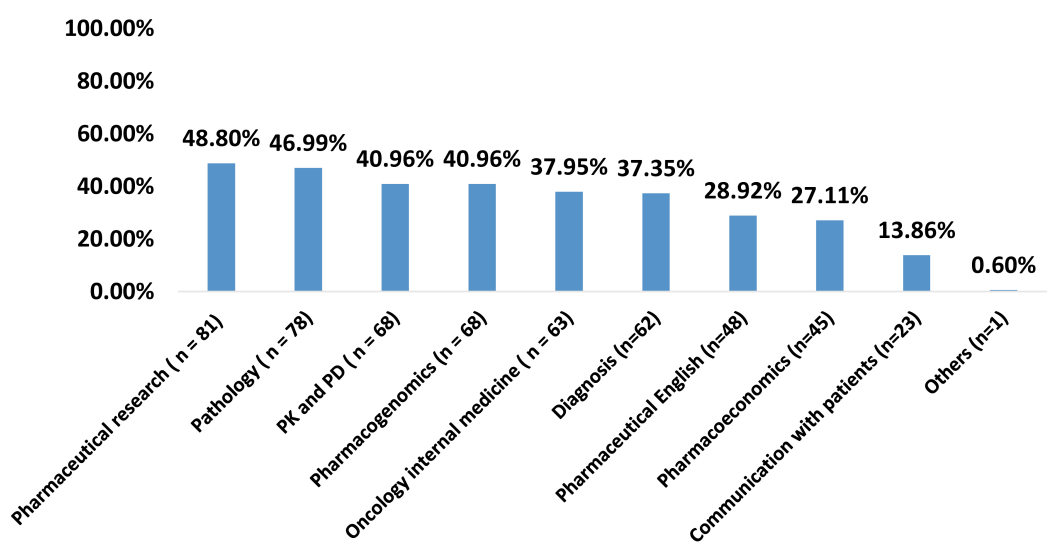

c

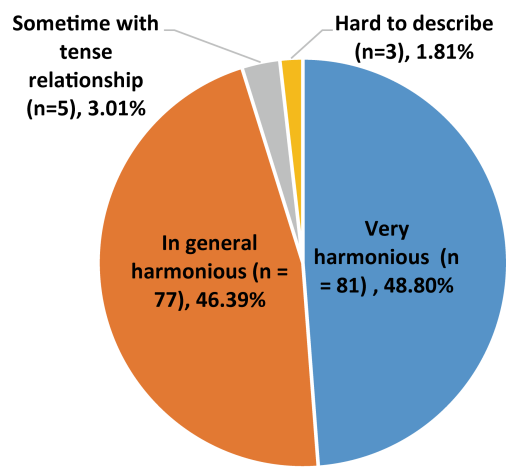

\section{Figure 4}

Self-evaluation of pharmacists on their services and relationship with other healthcare professionals. a. the pharmacotherapy problems that pharmacists were not good at (multiple choices). b. the disciplines that pharmacists thought they were relatively not good at but very necessary (multiple choices), PK: pharmacokinetics; PD: pharmacodynamics. c. the relationship between pharmacists and other healthcare professionals 

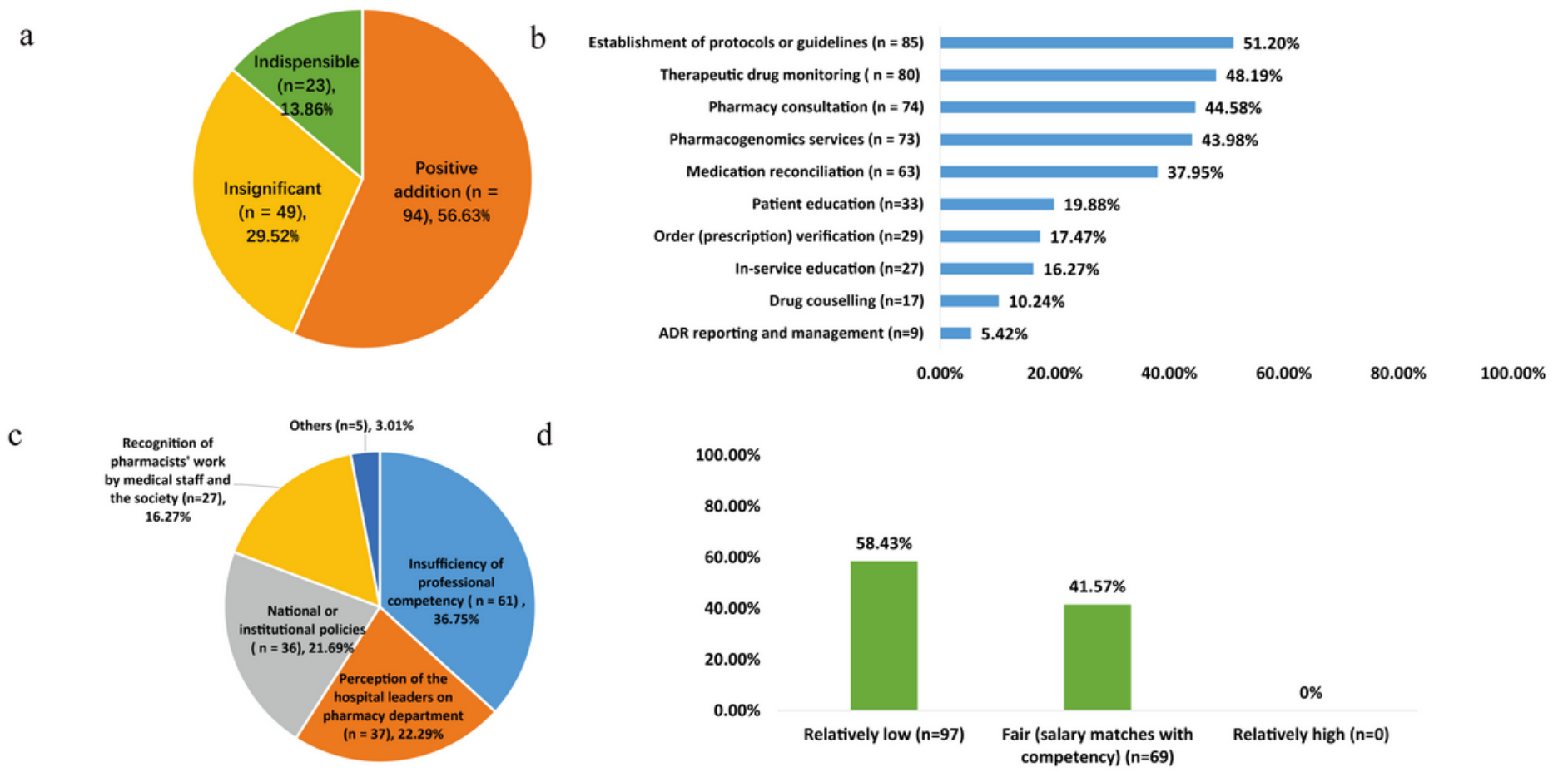

d

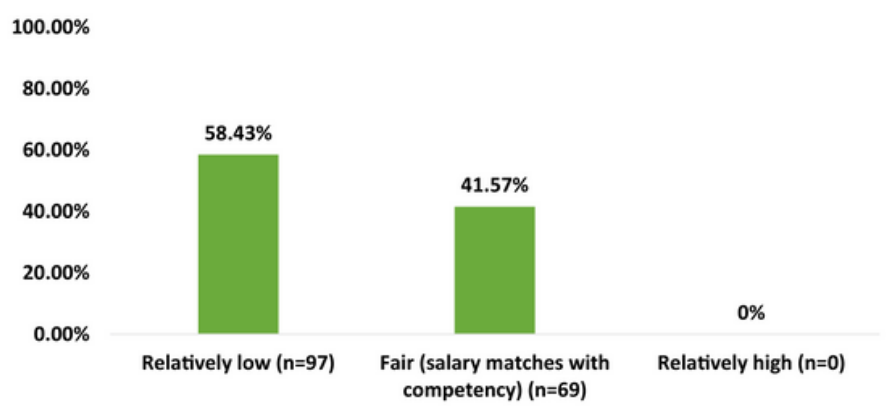

\section{Figure 5}

Self-evaluation of pharmacists on their services, salary and career development. a. pharmacists' selfevaluation on importance of their services to clinical departments. b. the categories of clinical pharmacy services considered by pharmacists to be inadequately performed (multiple choices). c. the response of pharmacists regarding "the biggest barrier for career development". $d$. the pharmacists' self-evaluation on their salary compared with their competency 